\title{
HD 49798: Its History of Binary Interaction and Future Evolution
}

\author{
Jared Brooks ${ }^{1}$, Thomas Kupfer $^{2}$ (1), and Lars Bildsten ${ }^{1,3}$ \\ ${ }^{1}$ Department of Physics, University of California, Santa Barbara, CA 93106, USA \\ 2 Division of Physics, Mathematics, and Astronomy, California Institute of Technology, Pasadena, CA 91125, USA \\ ${ }^{3}$ Kavli Institute for Theoretical Physics, University of California, Santa Barbara, CA 93106, USA \\ Received 2017 July 26; revised 2017 August 7; accepted 2017 August 7; published 2017 September 22
}

\begin{abstract}
The bright subdwarf-O star (sdO) HD 49798 is in a 1.55 day orbit with a compact companion that is spinning at $13.2 \mathrm{~s}$. Using the measurements of the effective temperature $\left(T_{\text {eff }}\right)$, surface gravity $(\log g)$, and surface abundances of the sdO, we construct models to study the evolution of this binary system using Modules for Experiments in Stellar Astrophysics (MESA). Previous studies of the compact companion have disagreed on whether it is a white dwarf (WD) or a neutron star (NS). From the published measurements of the companion's spin and spin-up rate, we agree with Mereghetti and collaborators that an NS companion is more likely. However, since there remains the possibility of a WD companion, we use our constructed MESA models to run simulations with both WD and NS companions that help us constrain the past and future evolution of this system. If it presently contains an NS, the immediate mass transfer evolution upon Roche lobe filling will lead to mass transfer rates comparable to that implied in ultraluminous X-ray sources (ULXs). Depending on the rate of angular momentum extraction via a wind, the fate of this system is either a wide ( $P_{\text {orb }} \approx 3$ day) intermediate-mass binary pulsar (IMPB) with a relatively rapidly spinning NS $(\approx 0.3 \mathrm{~s})$ and a high mass WD $\left(\approx 0.9 M_{\odot}\right)$, or a solitary millisecond pulsar (MSP).
\end{abstract}

Key words: binaries: close - stars: magnetars - subdwarfs - supernovae: general - X-rays: binaries

\section{Introduction}

HD 49798 is a binary system consisting of a hot and bright subdwarf in a 1.55 day orbit with a compact companion. At the time of its discovery and classification, it was the brightest hot subdwarf known (Jaschek \& Jaschek 1963), and remains one of the brightest today (Mereghetti et al. 2011). It was initially known to be a binary system, but Thackeray (1970) was the first to give a spectroscopic orbital period of 1.5477 days and to suggest that the compact companion may be a WD. Just two years later, Dufton (1972) performed a non-local thermodynamical equilibrium (LTE) analysis to derive estimates on the effective temperature $\left(T_{\text {eff }}\right)$ and the surface gravity $(\log g)$ of the sdO star, and Kudritzki \& Simon (1978) improved upon these measurements; based on non-LTE modeling, they found $T_{\text {eff }}=47,500 \pm 2000 \mathrm{~K}, \quad \log g=4.25 \pm 0.2, \quad y=50_{-7}^{+10} \%$, where $y=n(\mathrm{He}) / n$, as well as a projected rotational velocity of $v_{\text {rot }} \sin i=45 \pm 5 \mathrm{~km} \mathrm{~s}^{-1}$ for the hot subdwarf. This result was confirmed by an independent non-LTE analysis based on high-resolution Very Large Telescope (VLT)/UltravioletVisual Echelle Spectrograph (UVES) spectra (Müller 2009). $\mathrm{He}$ found $T_{\text {eff }}=46,500 \pm 500 \mathrm{~K}, \quad \log g=4.35 \pm 0.1$, $y=50 \%$, as well as a projected rotational velocity $v_{\text {rot }} \sin i=42 \pm 5 \mathrm{~km} \mathrm{~s}^{-1}$, which agrees with Kudritzki \& Simon (1978). Additionally, Bisscheroux et al. (1997) also did analysis on the subdwarf, and via a common envelope (CE), ejection efficiency parameterization concluded that an intermediate-mass star that entered into a $\mathrm{CE}$ while on the early AGB (EAGB) is the most likely progenitor to HD 49798.

This system was also detected in X-rays. Israel et al. (1995, 1996) published a detection of a $13.2 \mathrm{~s}$ period X-ray pulse, which is interpreted as the spin period of a magnetic compact companion accreting from the subdwarf wind. The estimates of the mass loss rates from the subdwarf, the capture rate onto the companion, and the associated accretion luminosity were compared to the observed X-ray luminosity by Israel et al.
(1996) and led to their suggestion that a neutron star (NS) was more likely than a WD.

The layout of this paper is as follows. We continue a short review of previous studies of this binary system and confirm that our sdO stellar model matches the observations in Section 2. Then in Section 3, we give our arguments for an NS companion and show results of binary modeling to give predictions on the future of the binary system. We also show results for binary modeling assuming a WD companion in Section 4. We explore the outcomes of a merger caused by a high rate of angular momentum loss via the system wind in Section 5, and finish with our conclusions in Section 6.

\section{Observational Analysis}

In this study, we build stellar models that match the measured values of $T_{\text {eff }}, \log g$, mass, and surface abundances of the sdO star and constrain the past and future evolution of this system using MESA version 8118 (Paxton et al. 2011, 2013, 2015).

\subsection{Previous Compact Object Interpretation}

Bisscheroux et al. (1997) looked at the same X-ray data from ROSAT as Israel et al. (1996), but used different estimates for the wind mass loss rates from the sdO star and concluded that a WD is more likely, but, an NS cannot be ruled out. One of their arguments against an NS companion has to do with their low birthrate and the small likelihood of seeing such a system. This argument does not hold because in the alternative scenario, a WD companion would accrete enough mass to reach $M_{\mathrm{Ch}}$ and undergo an accretion-induced collapse (AIC), leaving a subdwarf and an NS.

Several papers from the same group have been published on this system in the past few years (Mereghetti et al. 2009, 2011, 2013, 2016). Mereghetti et al. (2009) detected an eclipse in the X-ray light curve with a period coincident with the 
Table 1

Comparing to Observations of the sdO

\begin{tabular}{lcc}
\hline \hline Observable & Observed & $1.50 M_{\odot}$ Model \\
\hline$T_{\text {eff }}(\mathrm{K})$ & $47,500 \pm 2000$ & 47,500 \\
$\log g\left(\mathrm{~cm} \mathrm{~s}^{-2}\right)$ & $4.25 \pm 0.2$ & 4.41 \\
Radius $\left(R_{\odot}\right)$ & $1.45 \pm 0.25$ & 1.25 \\
$\log$ Lum. $\left(L_{\odot}\right)$ & $3.90 \pm 0.15$ & 3.85 \\
$X_{\mathrm{He}, \text { surf }}$ & $0.78 \pm 0.07$ & 0.78 \\
\hline
\end{tabular}

spectroscopic period. This allowed them to derive the inclination of the system and a much more precise measurement of the masses in the system and found $M_{\mathrm{sdO}}=1.50 \pm 0.05$, $M_{\mathrm{CC}}=1.28 \pm 0.05$, where $M_{\mathrm{CC}}$ is the mass of the compact companion. They also use the eclipse duration to measure the size of the X-ray emitting region to be $\approx 10^{4} \mathrm{~km}$, which is more that two orders of magnitude larger than the blackbody radius they derive from the X-ray spectrum. Just as in previous studies, the authors used wind-capture accretion rates and compared them to the X-ray luminosity to help distinguish between an NS or a WD companion. Mereghetti et al. (2009, 2011, 2013) all favor a WD over an NS companion, but the new angular momentum and magnetic field analysis in Mereghetti et al. (2016) suggests that an NS companion is more likely. Wang \& Han (2010) and Liu et al. (2015) performed calculations for this system assuming a $\mathrm{C} / \mathrm{O}$ WD companion and concluded that it may be an SN Ia progenitor. We did not pursue such an interpretation for two reasons: (1) C/O WDs are not expected to form above $1.05 M_{\odot}$ Piersanti et al. (2014), and (2) we expect that shell carbon ignitions would transform $\mathrm{C} / \mathrm{O}$ WDs to $\mathrm{O} / \mathrm{Ne}$ before reaching $M_{\mathrm{Ch}}$ (see Section 4).

\section{2. sdO Modeling}

Using MESA, we took the $1.5 M_{\odot}$ measurement of the mass of the subdwarf derived from the combination of the X-ray mass function and the optical mass function by Mereghetti et al. (2009) and constructed a model starting with a $7.15 M_{\odot}$ zero-age main-sequence (ZAMS) model, and started mass loss on the EAGB just before the second dredge-up, and ended mass loss when the surface helium mass fraction of the model matched the observed value.

When the effective temperature $\left(T_{\text {eff }}\right)$ of the subdwarf model reaches the observed $47,500 \mathrm{~K}$, the $\log g$ measurement, derived radius, and luminosity all agree with the model within the given error bars, as shown in Table 1. We also show this in the $T_{\text {eff }}-\log g$ diagram of Figure 1 .

At the time when the measurements match the model, the carbon core has grown to $0.71 M_{\odot}$, and the surface is blowing off a wind at $6 \times 10^{-9} M_{\odot} \mathrm{yr}^{-1}$, using the wind prescription from Bloecker (1995) and a scaling factor of 0.05. According to the model, the star will fill its RL approximately 65,000 years from now.

\section{NS Companion}

The measured spin-up rate of $\dot{P}=-2.15 \times 10^{-15} \mathrm{~s} \mathrm{~s}^{-1}$ given in Mereghetti et al. (2016) is high for a WD, requiring a relatively large accretion rate. At the maximum wind mass loss rate from the donor of $10^{-8} M_{\odot} \mathrm{yr}^{-1}$ (Hamann et al. 1981; Mereghetti et al. 2011), the companion would need to capture all the wind that crosses its RL to cause the measured spin up (see Equation (14) in Mereghetti et al. 2016), which is likely an overestimate of

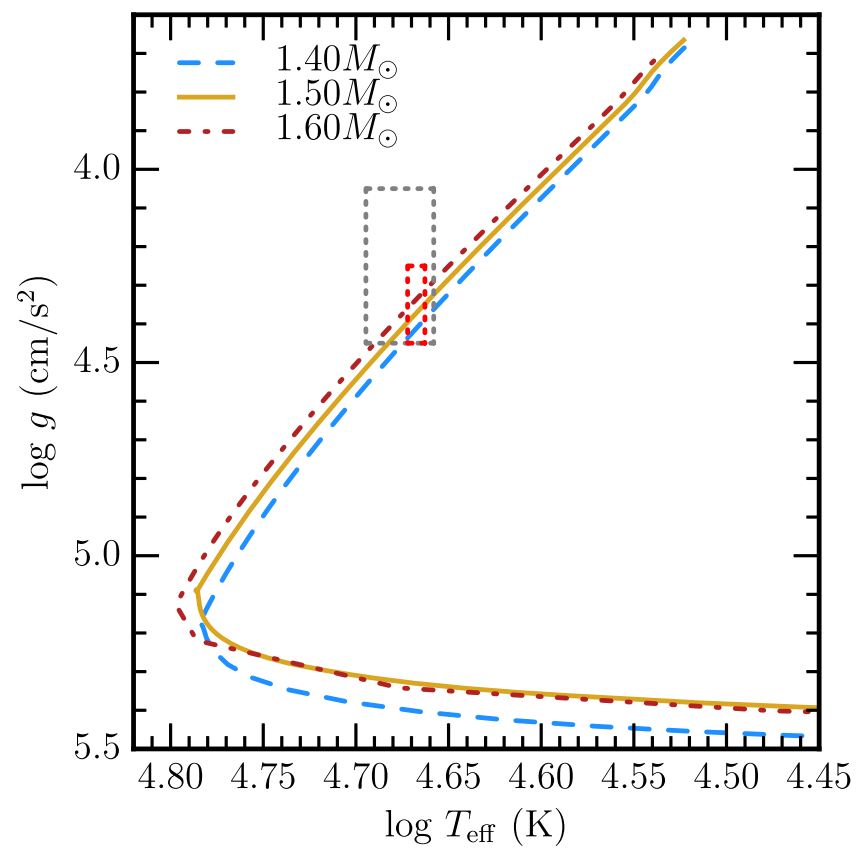

Figure 1. Dotted gray square represents the error box of the measurements of the $T_{\text {eff }}$ and $\log g$ of HD 49798 from Mereghetti et al. (2009), and the dotted red box is from Müller (2009). The curves show the evolution of helium cores of different masses evolving from the bottom of figure and move toward lower $T_{\text {eff }}$ and $\log g$ and are cut off as RLOF begins in the upper right of the plot.

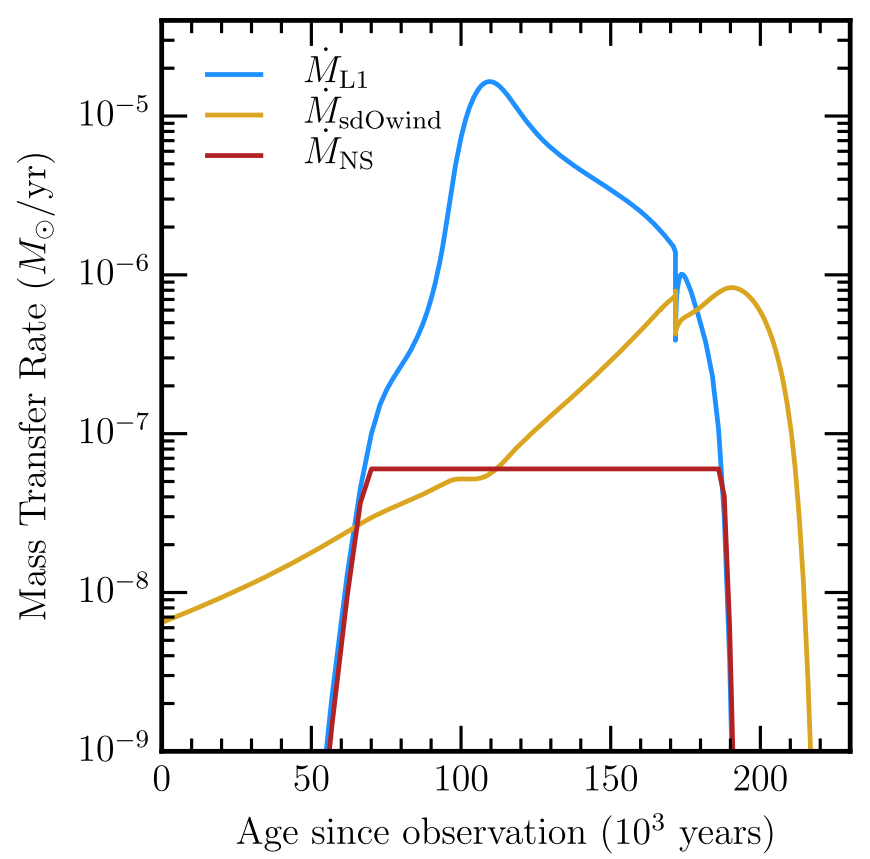

Figure 2. Blue line shows the rate of mass transfer via RLOF. The orange line shows the mass loss rate from the donor via winds. The dark red line shows the Eddington-limited mass gain rate of the NS.

the wind-capture rate. Therefore, we consider an NS interpretation of the compact companion to be much more likely.

If the companion is indeed an NS, there are two ways it could have formed: (1) via a core collapse supernova (CCSN) from a star with a ZAMS mass of $\gtrsim 10 M_{\odot}$, or (2) via AIC where the initially more massive star must have formed an $\mathrm{O} / \mathrm{Ne} \mathrm{WD}$ and subsequently accreted enough mass to reach $M_{\mathrm{Ch}}$. For the AIC progenitor scenario, the sdO star must have been RL filling at the moment of AIC and must be less than half RL filling just after the 
AIC to match the observations. Our calculations, based off the geometry of the system and the fact that the ejected mass from the AIC event (via neutrinos) takes with it the specific angular momentum of the $\mathrm{WD}$, find that the change in the $\mathrm{RL}$ radius from that mass and angular momentum loss would only be about $4 \%$. We ran models such that the donor was RL filling just before an AIC and is $4 \%$ below RL filling just after the AIC, with a 1.55 day orbital period. Since the donor had a deeply convective envelope and was therefore responding adiabatically to mass loss, the sudden shut-off of mass loss caused the star to shrink, but only to $16 \%$ below its RL before expanding to refill its RL, whereas we now observe it as about half-RL filling. If, however, the claimed distance of $650 \mathrm{pc}$ is an underestimate, then the radius of the sdO star would need to be significantly larger to match the measured $T_{\text {eff }}$ and luminosity simultaneously. The problems with this scenario is that the $T_{\text {eff }}, \log g$, and luminosity are already simultaneously matched for the model discussed in Section 3.1, implying that the radius is very near to what we show in Table 1 (Kudritzki \& Simon 1978), and this RL-filling progenitor model discussed here never matches the measured $T_{\text {eff }}$ and $\log g$.

Additionally, although small eccentricities are expected for most post-AIC systems, this applies to systems with orbital periods in the range from 10 to 50 days (Tauris 2015), whereas systems with periods of about a day end up in highly eccentric orbits (Chen et al. 2011). Given the uncertainties in tidal circularization timescales for these unusual binaries, however, we cannot say if the absence of an eccentricity provides any constraint on the origin of the compact object (Stickland \& Lloyd 1994; Mereghetti et al. 2011).

Furthermore, to achieve the measured surface $\mathrm{H}$ fraction in this scenario would require the fine tuning of having the AIC occur just as the last bit of the H-rich envelope was being transferred to the WD, whereas a $\mathrm{CE}$ removing only the $\mathrm{H}$-rich envelope and leaving just a little bit of surface $\mathrm{H}$ is a much more likely explanation. Therefore, if the companion is an NS, it was most likely formed via CCSN and not AIC.

\subsection{MESA Modeling of Binary}

We use MESA's binary module to evolve the model in a 1.55 day orbit with a $1.28 M_{\odot}$ point mass. In our calculations, we assume that the system wind takes with it the specific angular momentum of the companion. The high system wind rates during the Roche lobe overflow (RLOF) phase will be optically thick, assuming spherical symmetry, and reprocess any hightemperature accretion luminosity. If, however, our system-wind angular momentum assumption is a significant underestimate, then the high mass transfer rate experienced when the subdwarf begins RLOF could lead to a merger. We briefly explore the possible outcomes of a merger between the He star and a WD or NS companion in Section 5.

At RLOF the mass transfer rate through L1 quickly grows to $\approx 2 \times 10^{-5} M_{\odot} \mathrm{yr}^{-1}$, almost all of which is lost from the system due to the Eddington-limited accretion rate of the NS of $6 \times 10^{-8} M_{\odot} \mathrm{yr}^{-1}$ (Figure 2), meaning that the NS only gains $7.4 \times 10^{-3} M_{\odot}$ during this phase, which spins up the NS to a $33 \mathrm{~ms}$ spin period.

The system loses mass at such a high rate during RLOF that the wind becomes optically thick for $\approx 10^{4}$ years. We compute the radius at which the optical depth of the wind reaches unity assuming a spherically symmetric wind, a wind speed of the escape velocity of the companion's RL, and electron scattering opacity, then use that radius and Eddington luminosity of the

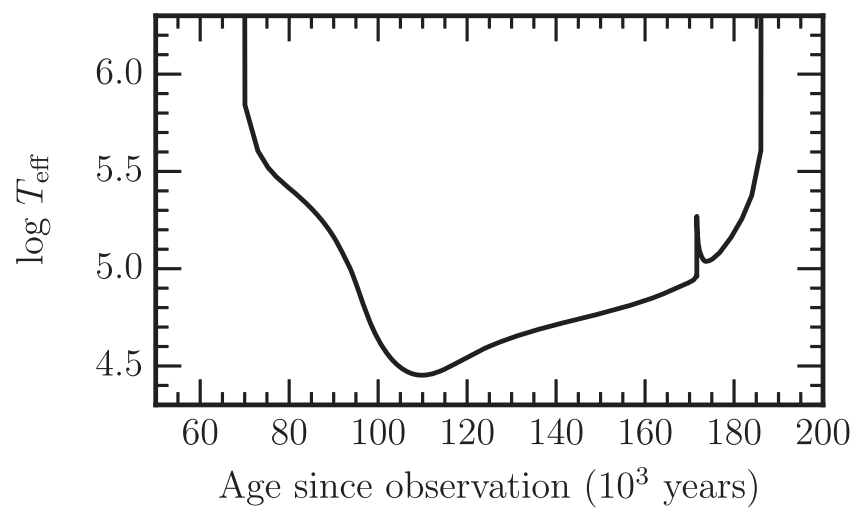

Figure 3. Effective temperature of the optically thick wind from the NS, assuming spherical symmetry, that reprocesses the thermal X-ray radiation to lower temperatures. We assume electron scattering opacity, a wind speed of the escape velocity of the NS's RL, and Eddington luminosity from the NS.

NS to compute the $T_{\text {eff }}$ that will be observed during the RLOF stage. As shown in Figure 3, the observed $T_{\text {eff }}$ from the wind decreases to $\approx 2.6 \times 10^{4} \mathrm{~K}$ at the highest mass loss rate.

When mass transfer completes and the donor star radius drops below the RL, the mass of the donor is $0.91 M_{\odot}$ and the orbital period is 2.7 days. At that orbital period, the inspiral time is much longer than the Hubble time, so the fate of this system is to be an intermediate-mass binary pulsar (IMBP) with the $0.91 M_{\odot} \mathrm{C} / \mathrm{O} \mathrm{WD}$ made from the He star.

\subsection{Possible ULX Source}

Ultraluminous X-rays (ULX) sources are powered by accreting NSs or stellar mass black holes at rates of $\approx 10^{-6} M_{\odot} \mathrm{yr}^{-1}$ (King et al. 2017). Recent detections of persistent pulsations (see Walton et al. 2017 for a summary) from many of these systems have proven that they often harbor an NS, implying that the accretion rate is $\gtrsim 10^{2}$ higher than the Eddington accretion rate. The binary evolution just described is a remarkable match for these ULX systems, as it stably provides accretion rates well above $10^{-6} M_{\odot} \mathrm{yr}^{-1}$ for a non-negligible amount of time $(\approx 80,000$ years) with an orbital period of 1.5 days. To explore the outcome of this type of ULX system, we ran an NS case where we set the maximum accretion rate of the NS to $\dot{M}_{\max }=10^{-6} M_{\odot} \mathrm{yr}^{-1}$. This does not qualitatively change the fate of the system (final donor star mass and orbital period after end of mass transfer are roughly the same) but the NS would reach an even more rapid rotation rate, up to $3 \mathrm{~ms}$ (rather than $33 \mathrm{~ms}$ ), due to the additional accreted material. This is a reasonable scenario for this accretion rate, as the estimated maximum magnetic field strength of $B_{s} \lesssim 8.9 \times 10^{9} \mathrm{G}$ (Mereghetti et al. 2016) would not allow for a magnetosphere to form outside the NS.

\section{WD Companion}

If we model the compact companion as a massive WD instead of an NS, the evolution of the He star is identical up until the start of RLOF, and extremely similar afterwards due to the mostly negligible difference in mass retention rates between the given scenarios. The maximum accretion rate of WDs is a few orders of magnitude higher than that for an NS, so a larger fraction of the mass donated by the He star remains in the system, but still more than half the mass is ejected (see Figure 4), taking with it the specific orbital angular momentum of the WD, as in Brooks et al. (2016), see also Wang \& Han 


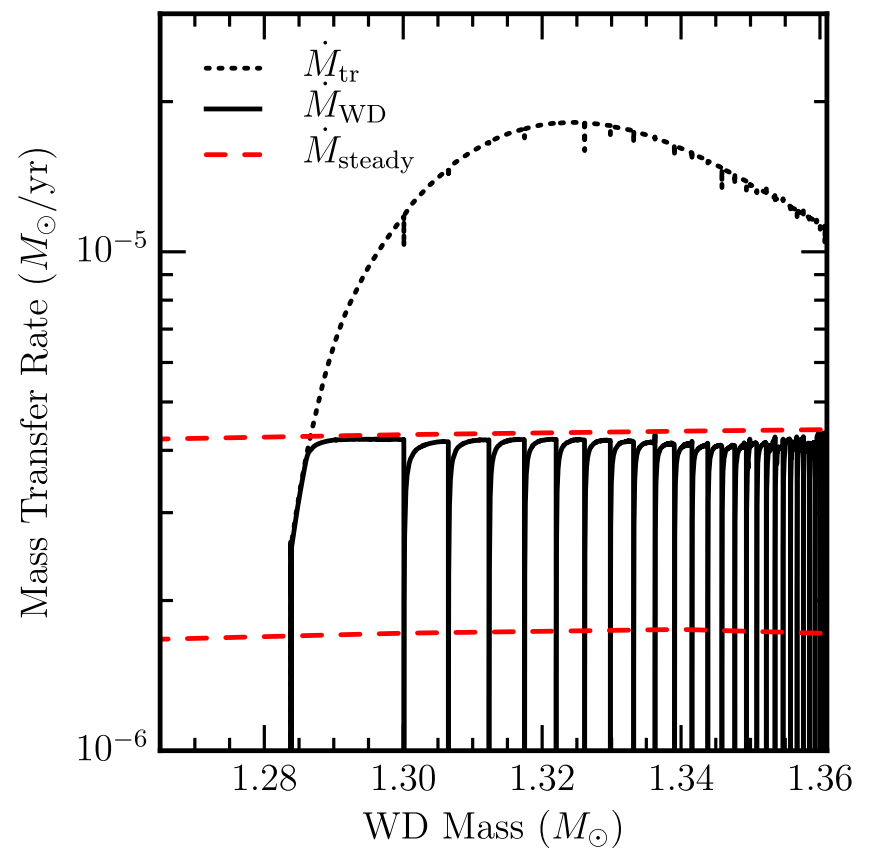

Figure 4. Mass transfer rate of a $1.28 M_{\odot} \mathrm{O} / \mathrm{Ne}$ WD in a 1.55 day orbital period binary system with a $1.5 M_{\odot}$ He star shown in solid black, which is punctuated by brief mass loss episodes caused by carbon flashes in the helium burning ashes. The solid tracks are the rate at which the WD is gaining mass; the dotted tracks are the rate at which the He star is losing mass. The difference between the dotted and solid track represents the mass that is lost from the system. The stable helium burning boundaries are shown by the dashed red lines from Brooks et al. (2016).

(2010) and Liu et al. (2015). The WD steadily burns He on the surface to $\mathrm{C} / \mathrm{O}$, building up hot $\mathrm{C} / \mathrm{O}$ layers that become unstable to runaway burning. The majority of energy released from carbon burning goes into neutrinos (which free stream out of the system) and increasing the entropy of the material, lifting the degeneracy of the $\mathrm{C} / \mathrm{O}$ layer and expanding the surface of the WD by a factor of $\approx 2$. This mild expansion is not enough to power mass loss, but is enough to temporarily prevent surface accretion. This can be seen in Figure 4, which shows the mass transfer rate by the dotted black line and the WD mass accretion rate by the solid black line, which has sharp dips caused by the carbon burning flashes (Brooks et al. 2017).

The WD grows in mass up to $1.36 M_{\odot}$ when electron captures at the center begin to remove pressure support, leading to a collapse of the WD into an NS (Nomoto 1987; Nomoto \& Kondo 1991; Woosley \& Baron 1992; Dessart et al. 2006; Schwab et al. 2015). At this stage, the He star has decreased in mass to $1.206 M_{\odot}$, and the period has increased to 1.9 days. The orbital period increases because, although the ejected mass carries away angular momentum, the conservation of angular momentum of the transferred mass from the donor to the accretor has the net effect of increasing the orbital period. The WD loses a significant amount of mass to neutrinos during the collapse to an NS, causing a sudden increase in the RL of the He star. The He star quickly refills its RL due to He shell burning, and the system closely resembles that of the start of the NS case (Section 3), with a smaller He star mass and a longer orbital period. When the mass transfer completes and the newly formed WD drops below the RL, the mass of the WD is $0.91 M_{\odot}$, which is the same as the NS scenario is Section 3, and the NS has gained $4.5 \times 10^{-3} M_{\odot}$. The final orbital period after the mass transfer completes is about 2.7 days, which is the same as the NS scenario; the inspiral time is much longer than the Hubble time, leaving this system as an IMBP.

Just as in the NS scenario in Section 3, the donor transfers mass much faster than the companion can accept it, so the system wind is optically thick and reprocesses the thermal X-ray radiation from the WD at $T_{\text {eff }} \gtrsim 10^{6} \mathrm{~K}$ to about $4 \times 10^{4} \mathrm{~K}$ at peak mass transfer rates.

\section{Merger Scenarios at RLOF}

For the simulations shown in Section 3.1, the system mass loss reaches $2 \times 10^{-5} M_{\odot} \mathrm{yr}^{-1}$. Since we assume that the system wind takes with it the specific angular momentum of the compact companion, the mass transfer is stable and leads to an overall increase in the orbital period and the binary separation. If, however, this is an underestimate of specific angular momentum of the system wind, the large system mass loss rates, thus the large angular momentum loss rates could lead to a merger.

As mass transfer rates rise due to helium shell burning in the donor, causing the star to rapidly expand into its RL, a large angular momentum loss rate will cause the size of the RL to shrink, increasing the mass loss rate, and will cause even faster angular momentum loss, resulting in runaway mass transfer. The helium from the donor would form a $\mathrm{CE}$, leading to a merger between the compact companion and the $0.71 M_{\odot} \mathrm{C} / \mathrm{O}$ core of the donor.

If the compact companion is an NS, then we can use the results from Metzger (2012) to predict the general outcome of such a merger. We use his model NS_C-O_1, as its parameters are similar to HD 49798. Using the wind rate and velocity from the disk and the total mass of the disrupted $\mathrm{C} / \mathrm{O}$ core $\left(0.71 M_{\odot}\right)$, we can estimate the total kinetic energy deposited by this disk wind. Comparing this to the binding energy of the helium, now in a $\mathrm{CE}$, we find that the energy from the disk wind is certainly large enough to eject all of the helium from the system. Most of the disk mass from the disrupted $\mathrm{C} / \mathrm{O}$ core is blown off in the disk wind, and only $\approx 0.11 M_{\odot}$ is deposited on the NS, according to estimates from the NS_C-O_1 model from Metzger (2012). This results in an NS of mass $M \approx 1.39 M_{\odot}$ with a spin period of $P_{\text {spin }} \approx 2.4 \mathrm{~ms}$, making this a millisecond pulsar of average NS mass.

If the compact companion is a WD, then there would be a similar lead up to the disruption of the $\mathrm{C} / \mathrm{O}$ core of the donor, but the remnant would last much longer. The disrupted $\mathrm{C} / \mathrm{O}$ sitting on top of the $1.28 M_{\odot} \mathrm{O} / \mathrm{Ne}$ core would go through a viscous phase and a carbon burning flame as outlined in Schwab et al. (2016), but since the WD would be primarily O/ $\mathrm{Ne}$, the flame would quench once it reached the $\mathrm{O} / \mathrm{Ne}$ core, preventing the lifting of degeneracy of the core. After the $\mathrm{C} / \mathrm{O}$ burns to $\mathrm{O} / \mathrm{Ne}$ and becomes part of the degenerate core, the core mass grows above $M_{\mathrm{Ch}}$ and electron captures start to relieve pressure in the center (before reaching conditions for neon burning, see Schwab et al. 2016), leading to an AIC.

\section{Conclusions}

We have shown that the observations of the sdO star HD 49798 are well fit by a star born with a $7.15 M_{\odot}$ ZAMS mass that enters into a $\mathrm{CE}$ just before the second dredge-up on the early AGB to become a $1.50 M_{\odot}$ He star with a $10^{-2} M_{\odot}$ H-rich envelope. Furthermore, the observations of the compact companion's X-ray pulsations suggest that an NS interpretation 
is more likely, but that a WD interpretation cannot be ruled out. We used MESA to simulate the evolution of this system, and predict that, for either an NS or WD companion, the fate of this system is for it to become a wide IMBP with a high mass $\left(\approx 0.9 M_{\odot}\right)$ WD and a relatively rapidly spinning NS. This result assumes that the system wind takes with it the specific angular momentum of the compact companion. If, however, the system wind extracts extra angular momentum, then the high mass transfer rates $\left(2 \times 10^{-5} M_{\odot} \mathrm{yr}^{-1}\right)$ can lead to mergers (see Section 5). In the event of a merger, whether the companion is a WD or NS, the predicted fate would be for it to become a solitary NS, which would be an MSP in the case of an NS companion. In the case of a WD companion going into a merger, the resulting spin period after an AIC inside of an extended helium envelope is uncertain.

If the companion is an NS, then we have shown that during accretion, the system may have properties consistent with an ULX. So far only two ULX systems have a confirmed companion. P13 has a confirmed blue supergiant donor of spectral type B9Ia (Motch et al. 2014). The accretor in P13 shows $\mathrm{a} \approx 0.42 \mathrm{~s}$ slowly spinning up period that demonstrates that the accretor in P13 is an NS (Fürst et al. 2016; Israel et al. 2017). The second known system, M101 ULX-1, has a Wolf-Rayet star donor in an 8.2 day orbit. The accretor is most likely a stellar mass black hole (Liu et al. 2013). Because of the lack of confirmed donor stars in ULX an sdO donor cannot be excluded in other ULXs. Therefore, we conclude that HD 49798 is a plausible progenitor binary to a ULX.

We thank Jim Fuller, Sterl Phinney, and Josiah Schwab for their helpful conversations. This research is funded by the Gordon and Betty Moore Foundation through Grant GBMF5076, and was also supported by the National Science Foundation under grant PHY 11-25915. We acknowledge stimulating workshops at Sky House where these ideas germinated.

\section{ORCID iDs}

Thomas Kupfer (iD https://orcid.org/0000-0002-6540-1484

\section{References}

Bisscheroux, B. C., Pols, O. R., Kahabka, P., Belloni, T., \& van den Heuvel, E. P. J. 1997, A\&A, 317, 815

Bloecker, T. 1995, A\&A, 297, 727

Brooks, J., Bildsten, L., Schwab, J., \& Paxton, B. 2016, ApJ, 821, 28

Brooks, J., Schwab, J., Bildsten, L., Quataert, E., \& Paxton, B. 2017, ApJ, 843,151

Chen, W.-C., Liu, X.-W., Xu, R.-X., \& Li, X.-D. 2011, MNRAS, 410, 1441

Dessart, L., Burrows, A., Ott, C. D., et al. 2006, ApJ, 644, 1063

Dufton, P. L. 1972, MNRAS, 159, 79

Fürst, F., Walton, D. J., Harrison, F. A., et al. 2016, ApJL, 831, L14

Hamann, W.-R., Gruschinske, J., Kudritzki, R. P., \& Simon, K. P. 1981, A\&A, 104,249

Israel, G. L., Papitto, A., Esposito, P., et al. 2017, MNRAS, 466, L48

Israel, G. L., Stella, L., Angelini, L., et al. 1996, ApJ, 474, L53

Israel, G. L., Stella, L., Angelini, L., White, N. E., \& Giommi, P. 1995, IAUC, 6277,1

Jaschek, M., \& Jaschek, C. 1963, PASP, 75, 365

King, A., Lasota, J.-P., \& Kluzniak, W. 2017, MNRAS, 468, 59

Kudritzki, R. P., \& Simon, K. P. 1978, A\&A, 70, 653

Liu, D.-D., Zhou, W.-H., Wu, C.-Y., \& Wang, B. 2015, RAA, 15, 1813

Liu, J.-F., Bregman, J. N., Bai, Y., Justham, S., \& Crowther, P. 2013, Natur, 503,500

Mereghetti, S., La Palombara, N., Tiengo, A., et al. 2011, ApJ, 737, 51

Mereghetti, S., Palombara, N. L., Tiengo, A., et al. 2013, A\&A, 553, 46

Mereghetti, S., Pintore, F., Esposito, P., et al. 2016, MNRAS, 458, 3523

Mereghetti, S., Tiengo, A., Esposito, P., et al. 2009, Sci, 325, 1222

Metzger, B. D. 2012, MNRAS, 419, 827

Motch, C., Pakull, M. W., Soria, R., Grisé, F., \& Pietrzyński, G. 2014, Natur, 514, 198

Müller, S. 2009, Master's thesis, Univ. Erlangen-Nuremberg

Nomoto, K. 1987, ApJ, 322, 206

Nomoto, K., \& Kondo, Y. 1991, ApJL, 367, L19

Paxton, B., Bildsten, L., Dotter, A., et al. 2011, ApJS, 192, 3

Paxton, B., Cantiello, M., Arras, P., et al. 2013, ApJS, 208, 4

Paxton, B., Marchant, P., Schwab, J., et al. 2015, ApJS, 220, 15

Piersanti, L., Tornambé, A., \& Yungelson, L. R. 2014, MNRAS, 445, 3239

Schwab, J., Quataert, E., \& Bildsten, L. 2015, MNRAS, 453, 1910

Schwab, J., Quataert, E., \& Kasen, D. 2016, MNRAS, 463, 3461

Stickland, D. J., \& Lloyd, C. 1994, Obs, 114, 41

Tauris, T. M. 2015, e-print (arXiv:1501.03882)

Thackeray, A. D. 1970, MNRAS, 150, 215

Walton, D. J., Fuerst, F., Harrison, F. A., et al. 2017, e-print (arXiv:1705 10297)

Wang, B., \& Han, Z.-W. 2010, RAA, 10, 681

Woosley, S. E., \& Baron, E. 1992, ApJ, 391, 228 